\title{
Taxonomy of Some Swine Mycoplasmas: Mycoplasma suipneumoniae Goodwin et al. 1965, a Later, Objective Synonym of Mycoplasma hyopneumoniae Mare and Switzer 1965, and the Status of Mycoplasma flocculare Meyling and Friis 1972
}

\author{
DAVID L. ROSE,' JOSEPH G. TULLY,' and RUTH G. WITTLER $\dagger$ \\ Laboratory of Infectious Diseases, National Institute of Allergy and Infectious Diseases, Bethesda, \\ Maryland 20014,' and Department of Bacterial Diseases, Walter Reed Army Institute of Research, \\ Washington, D.C. 20012
}

\begin{abstract}
Two mycoplasmas, Mycoplasma hyopneumoniae Mare and Switzer 1965 and $M$. suipneumoniae Goodwin et al. 1965, have been named and described as etiological agents in enzootic pneumonia of pigs. Authentic cultures of strain 11, the type strain of $M$. hyopneumoniae, are no longer extant. Strain J, the type strain of $M$. suipneumoniae, is here proposed as the neotype strain of $M$. hyopneumoniae. Since the names $M$. hyopneumoniae and $M$. suipneumoniae are now based on the same type, they are objective synonyms. The name $M$. hyopneumoniae, being the earlier of the two, has priority and is therefore the correct name of the species. The name $M$. suipneumoniae is thus illegitimate as a later, objective synonym. Another swine isolate, $M$. flocculare, although sharing a number of biological features with strains of $M$. hyopneumoniae, is nevertheless serologically distinct not only from the latter but also from all of the other currently recognized species of Mycoplasma. It is thus confirmed here as a unique species. Biological and serological characterizations of the type and proposed neotype strains are given.
\end{abstract}

Independently and almost simultaneously, two species names were proposed for a mycoplasma causing pneumonia in pigs. The name Mycoplasma hyopneumoniae was validly published by Mare and Switzer in 1965 (18). The type strain (by monotypy) of $M$. hyopneumoniae is strain 11 (also known as VPP 11), a culture of which was deposited by Switzer in the American Type Culture Collection (ATCC) under the number 25617 . Shortly thereafter, the name $M y$ coplasma suipneumoniae was proposed by Goodwin et al. (13) for the organism causing enzootic pneumonia of pigs. Their designated type strain (strain $\mathrm{J}$ ) was deposited in the National Collection of Type Cultures (NCTC) under the number 10110 and subsequently in the ATCC under the number 25943. However, according to Minute 4 of the meeting of the International Subcommittee on the Taxonomy of Mycoplasmatales held in Bordeaux, France, in September 1974 (28):“ . . the culture of strain 11 now available from the American Type Culture Collection (ATCC 25617) is not the culture originally deposited by Switzer with the ATCC. The latter was lost because it could not be

† Present address: Bay Ridge, MD 21403. maintained serially in subculture (Ruth Wittler 1970 , personal communication) and it was subsequently replaced by another culture also obtained from Switzer. Since, however, the passage history of this culture did not include cloning by appropriate methods (12), it is doubtful whether it is identical with the strain on which the description of $M$. hyopneumoniae was based, and hence a neotype has to be designated. As strain $J$, the type by monotypy of $M$. suipneumoniae $(2,4)$, is easier to grow and also otherwise more workable than strain 11 , from which it is serologically indistinguishable (Ruth Wittler 1973, unpublished data), the Subcommittee recommended that strain J (ATCC 25934, NCTC 10110) be accepted as the neotype of $M$. hyopneumoniae. It was agreed that a proposal to that effect should be published." Pursuant to this recommendation, one of the purposes of this paper is to propose strain $\mathrm{J}$ as the neotype strain of $M$. hyopneumoniae.

This report also documents data, part of which was supplied earlier to the International Committee on Systematic Bacteriology (ICSB) Subcommittee, obtained in a collaborative study on the biological and serological characteristics of cultures of strains 11 and $J$ maintained by the 
ATCC. During this study, another swine organism, Mycoplasma flocculare, was isolated, named, and described by Friis and Meyling (12, 22 ). This organism exhibited a number of biochemical characteristics that were very similar to those described for strains $\mathrm{J}$ and 11 . In addition, the type strain of $M$. flocculare (strain $\mathrm{Ms}$ 42) was not at that time compared with all of the then-described Mycoplasma species. It was, therefore, deemed useful to include strain Ms 42 in this comprehensive serological analysis of strains $\mathrm{J}$ and 11 and to assess their serological and biological interrelationships, as well as to enlarge the descriptions of each of the organisms involved in this study.

\section{MATERIALS AND METHODS}

Mycoplasma strains and cloning procedures. M. hyopneumoniae 11 (= ATCC 25617), M. suipneumoniae J (= ATCC 25934), and $M$. flocculare Ms 42 (= ATCC 27399) were obtained from the ATCC. Each culture was purified again by filtration-cloning procedures. Five milliliters of a broth culture was mixed with an equal quantity of sterile broth and filtered through a 450 -nm membrane filter. Serial 10-fold dilutions of the filtrate were made in broth, and at least three dilutions were plated onto agar. Plates were incubated in a candle jar at $37^{\circ} \mathrm{C}$ and examined daily for mycoplasma colonies. Isolated colonies were picked from plates containing not more than 10 colonies, and the organisms were reinoculated into broth medium. The filtration-cloning procedure was repeated twice more. Thrice filter-cloned cultures (designated FCX3) were compared serologically with the original cultures by growth inhibition tests before being characterized. The FCX 3 lines were also deposited in the ATCC as strain 11 (ATCC 27714), strain J (ATCC 27715), and strain Ms 42 (ATCC 27716).

Culture media. The three swine mycoplasmas were cultivated on a medium essentially adapted from the Cambridge A26 liquid medium (14). This medium contained the following ingredients: Hanks balanced salt solution (10X), without $\mathrm{NaHCO}_{3}$ or phenol red (Grand Island Biological Co. [GIBCO], Grand Island, N.Y.), $4 \mathrm{ml}$; Hartley digest broth (Oxoid Ltd., London, England), $30 \mathrm{ml}$; lactalbumin hydrolysate (Nutritional Biochemicals Corp., Cleveland, Ohio), $10 \mathrm{ml}$ of a $5 \%$ (wt/vol) solution in Dulbecco phosphate-buffered salt solution (GIBCO); fresh yeast extract (Microbiological Associates, Bethesda, Md.), $2 \mathrm{ml}$; porcine serum (GIBCO), $20 \mathrm{ml}$ of serum which had been acid-treated (29); $0.25 \%$ aqueous solution of phenol red, $1 \mathrm{ml}$; and deionized water, $36 \mathrm{ml}$. The final $\mathrm{pH}$ was 7.4. The complete broth medium was sterilized by filtration through a sterile Seitz S3 filter pad. Agar medium was prepared by adding $0.7 \%$ Ionagar no. 2 (Oxoid) to the deionized water component, sterilizing the agar base by heat, and then adding filter-sterilized broth components to the melted agar. Phenol red indicator was omitted from the agar.

Two other medium formulations were also employed in this study. The FF medium of Friis (10) was slightly modified for optimal cultivation of all three swine mycoplasmas. This medium consisted of the following: Hanks balanced salts (10X) (Flow Laboratories, Rockville, Md.), $30 \mathrm{ml}$; deionized water, $720 \mathrm{ml}$; brain heart infusion (Difco Laboratories, Detroit, Mich.), 5 g; mycoplasma broth base (BBL Microbiology Systems, Cockeysville, Md.), 7.5 g; lactalbumin hydrolysate (Nutritional Biochemicals Corp.), $1.25 \mathrm{~g}$; yeast extract (Difco), $0.5 \mathrm{~g}$; and $0.1 \%$ solution of phenol red, $13.7 \mathrm{ml}$. This base medium was adjusted to $\mathrm{pH} 7.8$ and sterilized at $15 \mathrm{lb} / \mathrm{in}^{2}\left(121^{\circ} \mathrm{C}\right)$ for $20 \mathrm{~min}$. The following sterile supplements were added aseptically: $25 \%$ solution of fresh yeast extract (Microbiological Associates), $36.5 \mathrm{ml}$; $50 \%$ solution of glucose, $2.5 \mathrm{ml}$; thallium acetate (1:50 solution), $5.5 \mathrm{ml}$; horse serum (Flow Laboratories), $100 \mathrm{ml}$; and porcine serum (GIBCO) that had been heat-inactivated at $56^{\circ} \mathrm{C}$ for $30 \mathrm{~min}, 100 \mathrm{ml}$; Ionagar no. 2 (Oxoid) was used in the base $(6.0 \mathrm{~g} /$ liter $)$ for a solid medium. The SP-4 medium for spiroplasmas (31) was used in several trials for ability to support the growth of the fastidious strains $\mathrm{J}$ and 11. This medium was modified by substitution of acid-treated porcine serum for the bovine serum component, and penicillin was omitted from all preparations.

Biochemical tests. An agar vial method (1) was used to test for utilization of carbohydrates, arginine, and urea. Mycoplasma broth cultures maintained in the A26 medium were incubated for 2 to 3 days at $37^{\circ} \mathrm{C}$ and were then centrifuged at $3,900 \times \mathrm{g}$. The sediment was suspended to the same volume in conventional mycoplasma broth medium containing $10 \%$ horse serum (1). The inoculum for the biochemical tests consisted of this suspension and test medium at a ratio of $0.7: 1 \mathrm{ml}$, respectively.

Phosphatase activity, serum digestion, and tetrazolium and tellurite reductions were also determined by the methods of Aluotto et al. (1). An agar overlay technique (34) was used to test for tetrazolium reduction and was adapted for tellurite-reduction detection by using a $0.1 \%$ solution of potassium tellurite in place of tetrazolium. The film-and-spot reaction was determined on heart infusion agar (Difco) supplemented with $10 \%$ heated horse serum, $10 \%$ concentrated egg yolk emulsion (Consolidated Labs, Glenwood, Ill.), and $10 \%$ fresh yeast extract (Microbiological Associates). Hemadsorption of sheep and guinea pig erythrocytes to mycoplasma colonies was determined by the method of Manchee and Taylor-Robinson (17). Mycoplasma sterol requirements were determined indirectly by sensitivity to $1.5 \%$ digitonin (9) and directly by growth determinations on agar media containing various quantities of cholesterol, fatty acids, etc. (8). Growth at 25 and $45^{\circ} \mathrm{C}$ was determined by incubating broth cultures at these temperatures, with plate counts performed on agar at zero time and after incubation for 7 and 14 days. Inhibition of mycoplasma growth by penicillin $\mathrm{G}$ (benzylpenicillin $K$ ) and erythromycin was assayed by incubating mycoplasmas in antibioticcontaining broth cultures for 14 days at $37^{\circ} \mathrm{C}$. Platings onto agar for determination of viability were made after $0,2,7$, and 14 days. Penicillin $G$ was tested at 10 and $100 \mathrm{IU} / \mathrm{ml}$, and erythromycin was tested at 10 and $100 \mu \mathrm{g} / \mathrm{ml}$. 
Filtration characteristics. Organisms from 3-dayold broth cultures (A26 medium) of each of the ATCC lines of strains J, 11, and Ms 42 were examined for their ability to pass through a series of membrane filters with graded porosities (450-, 300-, and $220-\mathrm{nm}$ average pore diameters). The original broth culture and each of the filtrates from the membrane filtrations were serially diluted (10-fold) in the same medium, and measured amounts of appropriate dilutions were plated to A26 (modified) agar medium. Agar plates were incubated in a candle jar $\left(37^{\circ} \mathrm{C}\right)$, and colony counts were performed after 10 to 14 days of incubation.

Antisera preparation. Antisera to each of the three swine mycoplasmas received from the ATCC were prepared in two rabbits. Antigens grown in $\mathbf{5 0 0}$ ml of culture medium (modified A26 medium with $20 \%$ uninactivated donkey serum from Flow Laboratories) were harvested by centrifugation at $23,000 \times g$ and washed twice in phosphate-buffered saline ( $\mathrm{pH} 7.5$ ); the pellets were then resuspended in $3.5 \mathrm{ml}$ of phosphate-buffered saline. An equal quantity of Freund complete adjuvant was added to each antigen. Each rabbit received approximately $3.2 \mathrm{ml}$ of the antigenadjuvant mixture at multiple tissue sites (intramuscularly in the shoulder and intradermally in the back and in the footpad of each hind leg). At 3-week intervals, test bleedings were obtained, and booster antigens were given until 5- to 6-mm zones of growthinhibiting antibody were observed, which generally required at least three booster doses and an immunization period of 60 days.

Hyperimmune antisera used in the heterologous serological comparisons of strains $\mathrm{J}$ and $\mathrm{Ms} 42$ were obtained from the collection of reference mycoplasma antisera maintained in the Mycoplasma Section of the Laboratory of Infectious Diseases, National Institute of Allergy and Infectious Diseases, Bethesda, Md.

Serological tests. Conventional growth inhibition tests (6) were performed with antisera prepared to the ATCC lines of strains $J, 11$, and Ms 42 against antigens obtained from both the primary ATCC cultures and the FC3X lines of each of these mycoplasmas. In several instances, antisera prepared elsewhere to strain $\mathrm{J}$ (obtained from $\mathrm{P}$. Whittlestone) and to strain Ms 42 (obtained from N. F. Friis) were also employed in growth inhibition tests. These tests were carried out on modified A26 agar medium, and plates were incubated at $37^{\circ} \mathrm{C}$ in candle jars.

A direct fluorescent-antibody test on mycoplasmas grown on agar plates was performed by a technique previously described (7). Fluorescein-conjugated antisera to each of the swine mycoplasmas were tested in homologous and heterologous comparisons, using twofold dilutions of each conjugate until end points of the fluorescent-antibody test system were determined.

The three swine mycoplasmas were also compared in growth inhibition and plate fluorescent-antibody tests with an extensive collection of antisera to recognized Mycoplasma species (Table 1).

Polyacrylamide gel electrophoresis. Cultures of the three strains were grown in broth of the same composition (modified FF medium), the cells were sedimented by centrifugation, and the cell proteins were solubilized in phenol-acetic acid-water (2:1:0.5, $w t / \mathrm{vol} / \mathrm{vol}$ ). The extracts recovered were then run in polyacrylamide gels containing $5 \mathrm{M}$ urea and $35 \%$ acetic acid, by procedures previously described (24).

\section{RESULTS}

Growth and cultural requirements. The modified A26 medium employed here supported growth of all swine mycoplasmas examined in this study. Yields of $10^{7}$ to $10^{8}$ colony-formingunits (CFU)/ml were obtained in liquid media after 2 to 3 days of incubation at $37^{\circ} \mathrm{C}$, and growth eventually reached $10^{10}$ to $10^{11} \mathrm{CFU} / \mathrm{ml}$ by 10 to 14 days. Colonies appeared on solid medium of this formulation after 5 to 7 days of incubation at $37^{\circ} \mathrm{C}$ in a candle jar containing approximately $5 \% \mathrm{CO}_{2}$. Colonies of strain $\mathrm{J}$ were larger than those of strain 11 on modified A26 medium, which facilitated several aspects of their serological analysis. The modified liquid FF medium of Friis also supported excellent growth of strains $J, 11$, and Ms 42, although the agar medium prepared from this formulation did not yield satisfactory colony growth after incubation under an aerobic or $5 \% \mathrm{CO}_{2}$ environment. Liquid medium SP-4, with an acid-treated porcine serum supplement, gave adequate growth $\left(10^{7}\right.$ to $\left.10^{8} \mathrm{CFU} / \mathrm{ml}\right)$ for strains $\mathrm{J}$ and 11 , but strain Ms 42 did not show suitable growth, even after 14 days of incubation.

All organisms examined grew best under aerobic conditions with supplements of $5 \% \mathrm{CO}_{2}$, as obtained with the candle jar. However, the organisms should be considered facultatively anaerobic rather than strictly aerobic, since growth occurred in broth and on agar when cultures were incubated at $37^{\circ} \mathrm{C}$ under various levels of anaerobiosis (including growth in an atmosphere of $95 \% \mathrm{~N}-5 \% \mathrm{CO}_{2}$ or a GasPak anaerobic system (BBL Microbiology Systems). Growth of organisms also occurred at $25^{\circ} \mathrm{C}$ in modified A26 medium, yielding $10^{7}$ to $10^{8} \mathrm{CFU} / \mathrm{ml}$ after 7 days and $10^{\circ} \mathrm{CFU} / \mathrm{ml}$ after 14 days of incubation. No growth was apparent at an incubation temperature of $45^{\circ} \mathrm{C}$.

Susceptibility to penicillin and erythromycin. The results of tests for susceptibility to penicillin $G$ and erythromycin for the three strains are shown in Table 2. Significant reductions in viable counts were apparent for all three mycoplasmas treated with 10 to $100 \mathrm{IU}$ of penicillin G per ml. No obvious susceptibility to erythromycin was noted for cultures containing this antibiotic after 14 days of incubation.

Biochemical and biological properties. Table 3 contains a summary of the biochemical and biological characteristics recorded for each of the strains under study. The three organisms 
TABLE 1. Mycoplasma strains and antisera used in serologic tests

\begin{tabular}{|c|c|c|c|}
\hline Species and strain & Source ${ }^{a}$ & Species and'strain & Source \\
\hline M. agalactiae PG2 & NIH & M. iners PG30 & NIH \\
\hline M. alkalescens DBS803 & MS-LID & M. lipophilum MaBy & NIH \\
\hline M. alvi Ilsley & MS-LID & M. maculosum PG15 & NIH \\
\hline$M$. anatis 1340 & NIH & M. meleagridis 17529 & NIH \\
\hline M. arginini G230 & NIH & M. moatsii MK405 & MS-LID \\
\hline M. arthritidis PG6 & NIH & M. molare $\mathrm{H} 542$ & MS-LID \\
\hline M. bovigenitalium PGi1 & NIH & M. mycoides subsp. mycoides B3 & MS-LID \\
\hline M. bovirhinis PG43 . . & NIH & M. mycoides subsp. capri PG3 & MS-LID \\
\hline$M$. bovis Donetta. . & MS-LID & M. neurolyticum type A $\ldots \ldots$ & NIH \\
\hline M. bovoculi M165/69 & MS-LID & M. opalescens MH5408 & MS-LID \\
\hline M. buccale CH 20247 & NIH & M. orale $\mathrm{CH} 19299$ & NIH \\
\hline M. canis PG14 & NIH & M. ovipneumoniae ST93 & MS-LID \\
\hline M. canadense $275 \mathrm{C}$ & MS-LID & M. pneumoniae $\mathrm{FH}$ & NIH \\
\hline M. capricolum California kid & MS-LID & M. primatum $\mathrm{HRC} 292$ & MS-LID \\
\hline M. caviae $\mathrm{G} 122$ & MS-LID & M. pulmonis PG34 & NIH \\
\hline M. citelli $\mathrm{RG}-2 \mathrm{C}$ & MS-LID & M. putrefaciens $\mathrm{KS}-1$ & MS-LID \\
\hline M. conjunctivae HRC581 & MS-LID & M. salivarium PG20 & NIH \\
\hline M. cynos $\mathrm{H} 831$ & MS-LID & M. spumans PG13 & NIH \\
\hline M. dispar $462 / 2$ & MS-LID & M. synoviae WVU1853 & MS-LID $^{b}$ \\
\hline M. edwardii PG24 & MS-LID & M. verecundum 107 & MS-LID \\
\hline M. equirhinis M432/72 & MS-LID & Mycoplasma sp. (primate) B291 & MS-LID \\
\hline M. faucium DC333. & NIH & Mycoplasma sp. (bovine) California calf & MS-LID \\
\hline M. feliminutum Ben & MS-LID & Mycoplasma (bovine group 7) B5P & MS-LID \\
\hline M. felis cat 27 & MS-LID & Mycoplasma sp. (bovine) 70-159 & MS-LID \\
\hline M. fermentans PG18 & NIH & Mycoplasma sp. (caprine) G145 & MS-LID \\
\hline M. flocculare Ms42 & MS-LID & Mycoplasma sp. (avian group C) CKK & MS-LID \\
\hline M. gallinarum PG16 & NIH & Mycoplasma sp. (avian group D) SA-J & MS-LID \\
\hline M. gallisepticum PG31 & NIH & Mycoplasma sp. (avian group F) WR1 & MS-LID \\
\hline M. gateae Mart . . & MS-LID & Mycoplasma sp. (avian group I) Iowa 695 & MS-LID \\
\hline$M$. hominis PG21 & NIH & Mycoplasma sp. (avian group L) $694 \ldots$ & MS-LID \\
\hline M. hyopneumonia $\mathrm{J}$ & MS-LID & & \\
\hline M. hyorhinis BTS7 & NIH & & \\
\hline M. hyosynoviae $\mathrm{S} 16$ & MS-LID & & \\
\hline
\end{tabular}

${ }^{a}$ Abbreviations: NIH, National Institutes of Health (NIAID-NIH research reference reagent); MS-LID, Mycoplasma Section, Laboratory of Infectious Diseases, National Institute of Allergy and Infectious Diseases, Bethesda, Md.

${ }^{b} M$. synoviae conjugated antiserum only used in serologic testing since growth-inhibiting serum was unavailable.

TABLE 2. Effect of penicillin $G$ (benzylpenicillin $K$ ) and erythromycin on growth of $M$. hyopneumoniae 11, M. suipneumoniae and $M$. flocculare Ms 42 in broth cultures of modified A26 medium

\begin{tabular}{|c|c|c|c|c|}
\hline \multirow{3}{*}{ Incubation period (days) } & \multicolumn{4}{|c|}{ Viable plate counts ${ }^{a}$ of broth medium containing: } \\
\hline & \multirow{2}{*}{ No antibiotic } & \multicolumn{2}{|c|}{ Penicillin G } & \multirow{2}{*}{ Erythromycin $(100 \mu \mathrm{g} / \mathrm{ml})$} \\
\hline & & $10 \mathrm{IU} / \mathrm{ml}$ & $100 \mathrm{IU} / \mathrm{ml}$ & \\
\hline \multicolumn{5}{|l|}{ M. hyopneumoniae 11} \\
\hline 0 & $5.3 \times 10^{5}$ & $3.7 \times 10^{5}$ & $4.7 \times 10^{5}$ & $4.3 \times 10^{5}$ \\
\hline 2 & $3.0 \times 10^{6}$ & $2.7 \times 10^{5}$ & $1.7 \times 10^{5}$ & $2.7 \times 10^{6}$ \\
\hline 7 & $6.7 \times 10^{8}$ & $3.3 \times 10^{5}$ & $2.3 \times 10^{5}$ & $8.3 \times 10^{8}$ \\
\hline 14 & $9.7 \times 10^{10}$ & $6.7 \times 10^{6}$ & $8.7 \times 10^{5}$ & $1.3 \times 10^{10}$ \\
\hline \multicolumn{5}{|l|}{ M. suipneumoniae $\mathrm{J}$} \\
\hline 0 & $6.7 \times 10^{5}$ & $8.3 \times 10^{5}$ & $7.3 \times 10^{5}$ & $7.7 \times 10^{5}$ \\
\hline 2 & $4.7 \times 10^{6}$ & $6.7 \times 10^{5}$ & $2.7 \times 10^{5}$ & $5.7 \times 10^{6}$ \\
\hline 7 & $8.7 \times 10^{8}$ & $7.3 \times 10^{5}$ & $4.3 \times 10^{5}$ & $1.3 \times 10^{9}$ \\
\hline 14 & $1.0 \times 10^{11}$ & $2.3 \times 10^{7}$ & $5.7 \times 10^{6}$ & $1.1 \times 10^{11}$ \\
\hline \multicolumn{5}{|l|}{ M. flocculare Ms 42} \\
\hline 0 & $1.0 \times 10^{5}$ & $9.7 \times 10^{4}$ & $1.0 \times 10^{5}$ & $1.3 \times 10^{5}$ \\
\hline 2 & $7.7 \times 10^{5}$ & $4.3 \times 10^{4}$ & $6.7 \times 10^{4}$ & $9.3 \times 10^{5}$ \\
\hline 7 & $6.7 \times 10^{7}$ & $4.7 \times 10^{4}$ & $8.3 \times 10^{4}$ & $4.7 \times 10^{7}$ \\
\hline 14 & $1.3 \times 10^{10}$ & $9.3 \times 10^{5}$ & $1.3 \times 10^{5}$ & $8.6 \times 10^{9}$ \\
\hline
\end{tabular}

\footnotetext{
${ }^{a}$ Expressed as CFU per milliliter of broth culture.
} 


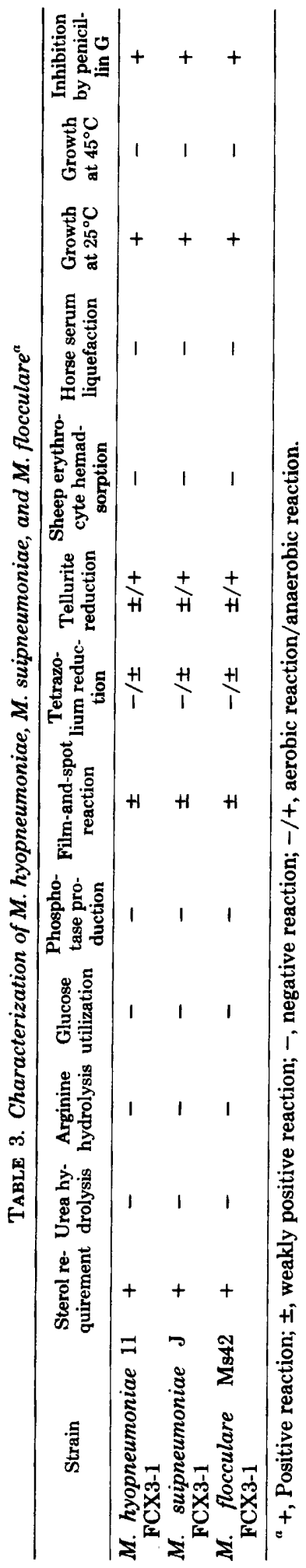

were found to require cholesterol in the agar plate method, since growth occurred only on media containing 10 to $20 \mu \mathrm{g}$ of cholesterol per $\mathrm{ml}$. These results were confirmed in tests to determine susceptibility to digitonin. Growth inhibition zones of 5.5 to $6.0 \mathrm{~mm}$ were observed around disks soaked with $1.5 \%$ digitonin solution which had been placed on agar plates seeded with dilutions (containing about $10^{4}$ to $10^{5} \mathrm{CFU} /$ $\mathrm{ml}$ ) of each of the three mycoplasmas.

The methods employed here did not indicate that any of the three mycoplasmas could utilize glucose or a number of other carbohydrates, including mannose, mannitol, arabinose, cellobiose, galactose, glycerol, inositol, fructose, salicin, sorbitol, sucrose, and xylose. Although a visible shift in $\mathrm{pH}$ indicator occurred in inoculated, modified A26 broth containing $0.5 \%$ glucose after an incubation period of 14 days, this shift did not exceed $0.5 \mathrm{pH}$ unit from that of inoculated control media lacking glucose or the other carbohydrates. Increases in growth were apparent in tubes containing carbohydrate; this observation was confirmed by selective plate counts and visible increases in turbidity. A test system based upon $\mathrm{pH}$ change was calculated as follows: (uninoculated substrate - inoculated substrate) - (uninoculated medium control inoculated medium control) $\geq 0.5$. In this system, the $\mathrm{pH}$ change with glucose varied from 0.2 to 0.4 unit, with an average change of 0.32 unit.

Filtration characteristics. The membrane filtration characteristics of each of the swine mycoplasmas examined are presented in Table 4. Passage of broth cultures through 450-nm filters decreased the viability titer by about $10^{5}$ $\mathrm{CFU} / \mathrm{ml}$. Only very small numbers of organisms were able to pass $300-\mathrm{nm}$ pore size filters, and no viable units were recovered in the $220-\mathrm{nm}$ filtrate.

Serological characteristics. A summary of results obtained from the growth inhibition and plate fluorescent-antibody serological tests with the strains under study is presented in Table 5. These comparisons included serological tests with the original ATCC lines and the FC3X cultures prepared for this study. Each of the lines of strain $J$ and strain 11 are serologically identical by both serological methods. Strain Ms 42 of $M$. flocculare did not exhibit any significant cross-relationships with strain J or 11. Strains J and Ms 42 were also compared with an extensive collection of reference antisera to recognized Mycoplasma species, subspecies, and a number of unclassified serotypes within this microbial group (Table 1) without any evidence that they were serologically related to any of these microorganisms.

Polyacrylamide gel patterns of cell proteins. The electrophoretic patterns in polyacryl- 
TABLE 4. Passage of three swine mycoplasmas through membrane filters

\begin{tabular}{lcccc}
\hline $\begin{array}{l}\text { Mycoplasma species and } \\
\text { strain }\end{array}$ & \multicolumn{2}{c}{ Viable plate counts $^{a}$ of broth cultures and filtrates after membrane filtration through: } \\
\cline { 2 - 5 } & No filtration & $\begin{array}{c}\text { 450-nm pore size } \\
\text { filter }\end{array}$ & $\begin{array}{c}\text { 300-nm pore size } \\
\text { filter }\end{array}$ & $\begin{array}{c}\text { 220-nm pore size } \\
\text { filter }\end{array}$ \\
\hline $\begin{array}{l}\text { M. hyopneumoniae 11 } \\
M \text {. suipneumoniae J }\end{array}$ & $7.7 \times 10^{9}$ & $5.3 \times 10^{4}$ & 3 & 0 \\
$M$. flocculare Ms 42 & $9.3 \times 10^{9}$ & $7.7 \times 10^{4}$ & 7 & 0 \\
\hline
\end{tabular}

a Counts expressed as CFU per milliliter of broth.

TABLE 5. Serological comparison of the type or proposed neotype strains of three swine mycoplasmas by growth inhibition and plate immunofluorescence antibody tests

\begin{tabular}{|c|c|c|c|c|c|c|}
\hline \multirow[b]{2}{*}{ Mycoplasma species and strain } & \multirow[b]{2}{*}{ ATCC no. } & \multicolumn{5}{|c|}{ Serological reactions ${ }^{a}$ with specific antisera or conjugates to strain: } \\
\hline & & $11^{b}$ (GI/FA) & $J^{c}(G I)$ & $\mathbf{J}^{b}(\mathrm{GI} / \mathrm{FA})$ & $\begin{array}{c}\operatorname{Ms~42^{d}} \\
\text { (GI) }\end{array}$ & $\begin{array}{c}\text { Ms 42 } \\
\text { (GI/FA) }\end{array}$ \\
\hline M. hyopneumoniae 11 & 25617 & $6.5 / 256^{e}$ & 5.0 & $5.0 / 256$ & $\sigma^{\prime}$ & $0 / 8$ \\
\hline M. hyopneumoniae $11(\mathrm{FC} \times 3)$ & 27714 & $6.5 / 256$ & 5.0 & $5.0 / 256$ & 0 & $0 / 8$ \\
\hline M. suipneumoniae $\mathrm{J}$ & 25934 & $6.5 / 256$ & 5.0 & $5.0 / 256$ & $\mathbf{0}$ & $0 / 8$ \\
\hline M. suipneumoniae J (FC $\times 3)$ & 27715 & $6.5 / 256$ & 5.0 & $5.0 / 256$ & 0 & $0 / 8$ \\
\hline M. flocculare Ms 42 & 27399 & $0 / 8$ & 0 & $0 / 8$ & 5.0 & $4.5 / 128$ \\
\hline$M$. flocculare Ms 42 (FC $\times 3)$ & 27716 & $0 / 8$ & 0 & $0 / 8$ & 5.0 & $4.5 / 128$ \\
\hline
\end{tabular}

${ }^{a}$ GI/FA, Growth inhibition/fluorescent-antibody tests.

${ }^{b}$ Antisera prepared by the Mycoplasma Section, Laboratory of Infectious Diseases, National Institute of Allergy and Infectious Diseases.

c Antiserum provided by P. Whittlestone, Cambridge, England.

${ }^{d}$ Antiserum provided by N. F. Friis, Copenhagen, Denmark.

e First number indicates zones of growth inhibition (in millimeters), and the second number indicates the reciprocal of the fluorescent-antibody titer.

$f$ Zero indicates no growth inhibition.

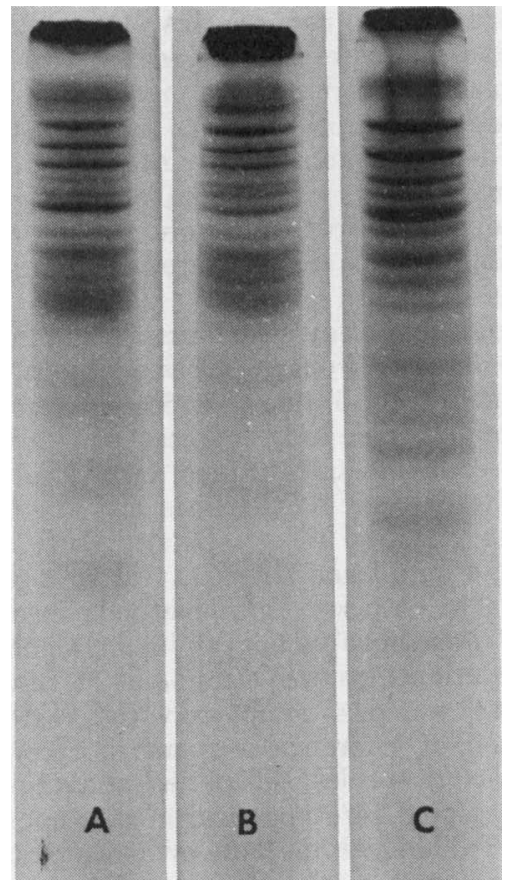

Fig. 1. Electrophoretic patterns of Mycoplasma cell proteins. (A) $M$. suipneumoniae $J$, (B) $M$. hyopneumoniae 11, and (C) $M$. flocculare $M s 42$. amide gels of the cell proteins of strains $\mathrm{J}, 11$, and Ms 42 are shown in Fig. 1. The patterns of strains $J$ and 11 are identical, which confirms the close serological and biological relationships established between these strains in this study. Although the gel pattern of strain Ms 42 of $M$. flocculare shows some similarities to the cell protein patterns of strains $\mathrm{J}$ and 11, there are some distinctive bands. Again, although the similarities to strains $\mathrm{J}$ and 11 probably relate to the close biochemical properties observed between strain Ms 42 and these two organisms, the differences correlate well with the recorded serological distinctions.

\section{DISCUSSION}

The observations recorded here indicate that strain 11 of $M$. hyopneumoniae and strain $\mathrm{J}$ of M. suipneumoniae, as maintained in the ATCC, are serologically and biologically indistinguishable. The data, therefore, substantiate earlier, but limited, information on the similarities between these two organisms recovered from pigs with enzootic pneumonia $(15,32)$.

The biological and biochemical properties presented here for these two strains supplement the description for the proposed taxonomic designation for this Mycoplasma species. Only one biochemical marker differs from those charac- 
teristics previously reported for these strains. On the basis of tests for utilization of glucose and other carbohydrates, we could not demonstrate that fermentation of carbohydrates occurred with either strain $J$ or strain 11 , as well as for strain Ms 42 of $M$. flocculare. This information conflicts with data reported earlier $(12,14,21$, 32). In light of these findings, we felt that a more critical study of the presence of hexokinases and various sugar transport enzymes (5) should be performed on each of these strains. Accordingly, a collaborative study with V. P. Cirillo has offered preliminary findings (unpublished data) suggesting that all three strains possess high levels of hexokinase activity in soluble cell fractions. However, the organisms do not appear to contain the sugar transport system found in most fermentative mycoplasmas (i.e., the phosphoenolpyruvate-dependent phosphotransferase system) (5). Additional studies are in progress to assess possible transport mechanisms and to measure uptake of radioactive glucose in actively metabolizing cultures of each of these strains. While this manuscript was in preparation, Jensen and associates (16) published data on the hexokinase activity of several strains of $M$. suipneumoniae and M. flocculare. They also stated that a recorded decline in glucose content of a broth medium (as measured by the glucose oxidase method) during growth of these strains was indicative of glucose metabolism. Although it is agreed that these organisms now possess hexokinases, we suggest that the glucose oxidase method is not a definitive method for determining glucose utilization. Other data will be required to establish transport or carrier mechanisms necessary to get glucose inside the mycoplasma cell, and this information will be required to define these organisms as fermentative or nonfermentative mycoplasmas. In addition, data along these lines may provide assurance that some serological procedures currently used with these organisms, such as the metabolism inhibition test in which a glucose substrate is employed, can be relied upon to measure specific antibody.

Observations on the inhibitory activity of benzylpenicillin toward the two Mycoplasma species studied here confirms earlier findings of Friis (11), although others have not observed this inhibition against some $M$. hyopneumoniae strains (33). Benzylpenicillin has also been noted to affect the growth and recovery of $M$. dispar (2) and $M$. neurolyticum (35). Benzylpenicillin in amounts of 10 to $100 \mathrm{IU} / \mathrm{ml}$ is not bactericidal to $M$. hyopneumoniae or $M$. flocculare but, as demonstrated in this study, significant inhibition in growth occurs over a 14-day incubation period. Since field specimens may, at times, contain small numbers of mycoplasmas, it would seem advisable not to use this antibiotic in primary isolation or maintenance media for both swine Mycoplasma species. Although the data reported here and elsewhere (23) indicate that erythromycin might be a suitable substitute for benzylpenicillin, reports of minimal inhibitory levels of 4.9 to $25.0 \mu \mathrm{g}$ of erythromycin per $\mathrm{ml}$ for $M$. hyopneumoniae are available $(30,33)$. Likewise, ampicillin, which has been recommended for use in isolation media for penicillinsusceptible strains of $\boldsymbol{M}$. dispar (2), has also yielded conflicting results in susceptibility tests with these swine mycoplasmas (N. F. Friis, $\mathrm{Ph}$.D. thesis, Royal Veterinary and Agricultural University, Copenhagen, Denmark, 1974; 33). Thus, although the use of benzylpenicillin in isolation media seems contraindicated, there is no clear consensus as to which antibiotic might be the least inhibitory to these swine mycoplasmas but still effective in suppressing other microbial agents during primary isolation under field conditions.

The serological separation of $M$. flocculare from $M$. hyopneumoniae would appear to be determined most effectively by the growth inhibition and plate fluorescent-antibody tests, providing antisera of adequate potency for each of the organisms are employed (22). We did not encounter problems previously described for the plate fluorescent-antibody test with these organisms (i.e., those primarily associated with the removal of colonies from agar surfaces by washing procedures [25]). It should be noted also that a newly described enzyme-linked immunosorbent assay procedure was able to distinguish $\boldsymbol{M}$. flocculare from $\boldsymbol{M}$. hyopneumoniae in pig lung preparations and in measurement of antibody responses in pig serum $(3,4)$.

The observations recorded here support the opinions expressed by the ICSB Subcommittee on the Taxonomy of Mycoplasmatales (28) that strain $\mathrm{J}$ is easier to work with, grows better on agar, and provides a more satisfactory test antigen for serological procedures. Strain J also possesses properties that are compatible with those originally described for $M$. hyopneumoniae. Although only the most meagre information on the biological characteristics of $M$. hyopneumoniae was supplied in the original publication (18), there is general agreement from data presented by Goodwin and associates (13-15) and from information in this report that the properties of strain $\mathrm{J}$ are similar to those described initially for $M$. hyopneumoniae, including morphology, lack of reversion to bacterial forms when the organisms are grown in antibiotic-free medium, and failure to pass filters with minimum pore diameters of $220 \mathrm{~nm}$ or less. Thus, strain $J$ qualifies in all respects as the proposed neotype strain of $M$. hyopneumoniae. In addition, the 
uncertain history of the original strain 11 would also constitute a point in favor of establishing strain $\mathrm{J}$ as the neotype of $M$. hyopneumoniae.

The extensive serological comparisons made between strain $J$ and strains of the other established Mycoplasma species used in this study have confirmed that the serological properties of this organism are distinct from those of all other currently recognized members of the genus $M y$ coplasma. The serological findings with $M$. flocculare provide substantial confirmation of the serological distinctions of this organism not only from $M$. hyopneumoniae strains but also from strains of other established Mycoplasma species. Finally, the serological and biological data obtained on strains $J$ and Ms 42 extend the taxonomic descriptions of these two organisms.

Thus, we propose strain $J$ as the neotype strain for $M$. hyopneumoniae. Because the names $M$. hyopneumoniae and $M$. suipneumoniae are based on the same type, they are objective synonyms. $M$. hyopneumoniae, being the earlier name, has priority over $M$. suipneumoniae, which is now illegitimate as a later, objective synonym. Furthermore, on the basis of a comparison of the proposed neotype strain of $M$. hyopneumoniae with the type strain of $M$. flocculare, it is evident that the two organisms are distinct species in the genus Mycoplasma.

\section{ACKNOWLEDGMENTS}

We thank P. Whittlestone and N. F. Friis for their assistance in supplying antisera for this study. We are also grateful to Colis Blood for technical aid during this work.

\section{REPRINT REQUESTS}

Address reprint requests to: D. L. Rose, Mycoplasma Section, Laboratory of Infectious Diseases, Building 7, Room 200, National Institute of Allergy and Infectious Diseases, Bethesda, MD 20014

\section{LTTERATURE CITED}

1. Aluotto, B. B., R. G. Wittler, C. O. Williams, and J. E. Faber. 1970. Standardized bacteriologic techniques for the characterization of Mycoplasma species. Int. J. Syst. Bacteriol. 20:35-58.

2. Andrews, B. E., R. H. Leach, R. N. Gourlay, and C. J. Howard. 1973. Enhanced isolation of Mycoplasma dis. par by substitution of ampicillin for benzylpenicillin in growth media. Vet. Rec. 93:603.

3. Bruggman, S., B. Engberg, and F. Ehrensperger. 1977. Demonstration of $M$. suipneumoniae in pig lungs by the enzyme-linked immunoperoxidase technique. Vet. Rec. 101:137.

4. Bruggmann, S., and H. Keller. 1977. Quantitative detection of antibodies to Mycoplasma suipneumoniae in pigs' sera by an enzyme-linked immunosorbent assay. Vet. Rec. 101:109-111.

5. Cirillo, V. P., and S. Raxin. 1973. Distribution of a phosphoenolpyruvate-dependent sugar phosphotransferase system in mycoplasmas. J. Bacteriol. 113:212217.
6. Clyde, W. A., Jr. 1964. Mycoplasma species identification based upon growth inhibition by specific antisera. J. Immunol. 92:958-965.

7. Del Giudice, R. A., N. F. Robillard, and T. R. Carski. 1967. Immunofluorescence identification of Mycoplasma on agar by use of incident illumination. J. Bacteriol. 98:1205-1209.

8. Edward, D. G. ff. 1971. Determination of sterol requirement for Mycoplasmatales. J. Gen. Microbiol. 69:205210.

9. Freundt, E. A., B. E. Andrews, H. Ernø, M. Kunze, and F. T. Black. 1973. The sensitivity of Mycoplasmatales to sodium polyanethol sulfonate and digitonin. Zentralbl. Bakteriol. Parasitenkd. Infektionskr. Hyg. Abt. 1 Orig. Reihe A 225:104-112.

10. Friis, N. F. 1971. Mycoplasmas cultivated from the respiratory tract of Danish pigs. Acta Vet. Scand. 12:6979.

11. Friis, N. F. 1971. Sensitivity of Mycoplasma suipneumoniae to penicillin G. Acta Vet. Scand. 12:120-121.

12. Friis, N. F. 1972. Isolation and characterization of a new porcine mycoplasma. Acta Vet. Scand. 13:284-286.

13. Goodwin, R. F. W., A. P. Pomeroy, and P. Whittlestone. 1965. Production of enzootic pneumonia in pigs with a mycoplasma. Vet. Rec. 77:1247-1249.

14. Goodwin, R. F. W., A. P. Pomeroy, and P. Whittlestone. 1967. Characterization of Mycoplasma suipneumoniae: a mycoplasma causing enzootic pneumonia in pigs. J. Hyg. 65:85-96.

15. Goodwin, R. F. W., and P. Whittlestone. 1966. Enzootic pneumonia of pigs: growth and behaviour of the casual mycoplasma in liquid media. Br. J. Exp. Pathol. 47: 518-524.

16. Jensen, P. T., C. Wolstrup, and N. F. Friis. 1978. Utilization of glucose by Mycoplasma suipneumoniae and Mycoplasma flocculare. Acta Vet. Scand. 19:179183.

17. Manchee, R. J., and D. Taylor-Robinson. 1968. Haemadsorption and hemagglutination by mycoplasmas. $\mathrm{J}$. Gen. Microbiol. 60:465-478.

18. Mare, C. J., and W. P. Switzer. 1965. Mycoplasma hyopenumoniae, a causative agent of virus pig pneumonia. Vet. Med. 60:841-846.

19. Mare, C. J., and W. P. Switzer. 1966. Virus pneumonia of pigs: drug and ether sensitivity of a causative agent. Am. J. Vet. Res. 27:1671-1675.

20. Mare, C. J., and W. P. Switzer. 1966. Virus pneumonia of pigs: filtration and visualization of a causative agent. Am. J. Vet. Res. 27:1677-1686.

21. Mare, C. J., and W. P. Switzer. 1966. Virus pneumonia of pigs: propragation and characterization of a causative agent. Am. J. Vet. Res. 27:1687-1693.

22. Meyling, A., and N. F. Friis. 1972. Serological identification of a new porcine Mycoplasma species, $M$. flocculare. Acta Vet. Scand. 13:287-289.

23. Ogata, M., H. Atobe, H. Kushida, and K. Yamamoto. 1971. In vitro sensitivity of mycoplasmas isolated from various animals and sewage to antibiotics and nitrofurans. J. Antibiot. 24:443-451.

24. Raxin, S., and S. Rottem. 1967. Identification of Mycoplasma and other microorganisms by polyacrylamidegel electrophoresis of cell proteins. J. Bacteriol. 94: 1807-1810.

25. Schuller, W., H. D. Lehmkuhl, and W. P. Switzer. 1976. A fluorescent antibody technique for identification of Mycoplasma hyopneumoniae colonies. Am. J. Vet. Res. 37:477-478.

26. Subcommittee on the Taxonomy of Mycoplasmatales. 1971. Minutes of the meeting, 10 August 1970. Int. J. Syst. Bacteriol. 21:151-153.

27. Subcommittee on the Taxonomy of Mycoplasmatales. 1974. Minutes of the meetings, 5 and 6 September 1973. Int. J. Syst. Bacteriol. 24:390-392. 
28. Subcommittee on the Taxonomy of Mycoplasmatales. 1975. Minutes of the meeting, 18 September 1974. Int. J. Syst. Bacteriol. 25:237-239.

29. Switzer, W. P. 1972. Mycoplasmal pneumonia of swine. J. Am. Vet. Med. Assoc. 160:651-653.

30. Takahashi, K., C. Kuniyasu, Y. Yoshida, and E. Momotani. 1978. Sensitivity in vitro to macrolide antibiotics and tetracyclines of Mycoplasma hyopneumoniae isolated from pneumonic porcine lungs. Natl. Inst. Anim. Health Q. 18:41-42.

31. Tully, J. G., R. F. Whitcomb, H. F. Clark and D. L. Williamson. 1977. Pathogenic mycoplasmas: cultivation and vertebrate pathogenicity of a new spiroplasma.
Science 195:892-894.

32. Whittlestone, P. 1973. Enzootic pneumonia of pigs (EPP). Adv. Vet. Sci. Comp. Med. 17:1-55.

33. Williams, P. P. 1978 . In vitro susceptibility of $M y c o$ plasma hyopneumoniae and Mycoplasma hyorhinis to fifty-one antimicrobial agents. Antimicrob. Agents Chemother. 14:210-213.

34. Woods, L. L., and T. F. Smith. 1972. Tetrazolium agar overlay in test for Mycoplasma pneumoniae. Appl. Microbiol. 24: 148-149.

35. Wright, D. N. 1967. Nature of penicillin-induced growth inhibition of Mycoplasma neurolyticum. J. Bacteriol. 93:185-190. 